\title{
Camrelizumab-related lethal arrhythmias and myasthenic crisis in a patient with metastatic thymoma: a case report
}

\section{Bo Zhang ( 13648403273@163.com )}

The Second Affiliated Hospital of Chongqing Medical University https://orcid.org/0000-0002-65715213

\section{Laxman Gyawali}

The Second Affiliated Hospital of Chongqing Medical University

Zengzhang Liu

The Second Affiliated Hospital of Chongqing Medical University

\section{Huaan Du}

The Second Affiliated Hospital of Chongqing Medical University

\section{Yuehui Yin}

The Second Affiliated Hospital of Chongqing Medical University

\section{Research Article}

Keywords: Immune checkpoint inhibitor, Immune related adverse event, Arrhythmia, Myasthenic crisis, Camrelizumab

Posted Date: March 18th, 2021

DOl: https://doi.org/10.21203/rs.3.rs-305090/v1

License: @ (i) This work is licensed under a Creative Commons Attribution 4.0 International License. Read Full License 
Camrelizumab-related lethal arrhythmias and myasthenic crisis in a patient with metastatic thymoma: a case report

Bo Zhang ${ }^{1}$, Laxman Gyawali ${ }^{1}$, Zengzhang Liu ${ }^{1}$, Huaan Du' ${ }^{1}$, Yuehui Yin ${ }^{1}$

1 Department of Cardiology, the Second Affiliated hospital of Chongqing Medical University, Chongqing, China

Corresponding author:

Huaan Du

Email: duhuaan20@126.com

Acknowledgement The authors would like to thank all relevant medical staff for the management of this patient.

\section{Declarations}

Funding No funding was received for this study.

Conflicts of interest All authors declare that they have no conflicts of interest.

Availability of data and material Data of the current publication are available from the corresponding author on reasonable request.

Code availability Not applicable.

Authors' contributions Material preparation, data collection and analysis were conducted by Bo Zhang, Laxman Gyawali, Zengzhang Liu, Huaan Du and Yuehui Yin. The first draft of the manuscript was written by Bo Zhang and all authors commented on the previous version. All authors approved the final manuscript.

Ethics approval All procedures performed in this study involving human participants were in accordance with the Helsinki and ethical standards of the 
institutional and/or national research committee.

Consent to participate Not applicable.

Consent for publication Consent for publication of the study was obtained from family members of the patient. 


\section{Summary}

Immune checkpoint inhibitors (ICIs) have emerged in recent years as a promising treatment option for several malignant tumors. However, ICI therapy has also been associated with various immune-related adverse events (irAEs), especially with pre-existing autoimmune status, which sometimes can be life-threatening. A 68-year-old woman diagnosed with metastatic thymoma was treated with camrelizumab as her initial anti-tumor protocol at a nearby hospital. On 11 days after the first dose of camrelizumab, the patient was admitted to our hospital with symptoms of dyspnea, fatigue, and poor appetite. Workup on admission indicated dramatically elevated transaminase, troponin I, creatine kinase, and creatine kinase MB and a new-onset conduction abnormality on electrocardiography. She had no other underlying disease prior to ICI treatment; therefore, ICI-related myocarditis, myositis and hepatitis were diagnosed, and intravenous methylprednisolone $(80 \mathrm{mg} /$ day $)$ and other supporting treatments were administered sequentially. Coronary angiography was performed on day 3 of hospitalization, but no abnormality was detected. On the same day, she lapsed into a coma with respiratory muscle failure, which was highly suspected of myasthenic crisis. Therefore, mechanical ventilation and higher dose of methylprednisolone ( $1 \mathrm{~g} /$ day) plus intravenous immunoglobulin (20g/day) were applied immediately. The third artrioventricular block occurred abruptly and an urgent temporary pacemaker was placed. Repeated ventricular tachycardia (VT) occurred, and even multiple antiarrhythmic drugs used in combination failed to alleviate the VT storm. On day 5 of hospitalization, she suffered 
from ventricular fibrillation and die of cardiac arrest. In conclusion, close follow-up should be conducted after ICI treatment, especially for patients already with or at high risk for autoimmune disorders. Once diagnosed with severe irAEs, prompt high dose of glucocorticoid alone or in combination with other immunomodulators if necessary should be administered. A multidisciplinary team approach is of importance for better management of patients with multiple organs involvement.

Keywords Immune checkpoint inhibitor - Immune-related adverse event $\cdot$ Arrhythmia $・$ Myasthenic crisis $・$ Camrelizumab 


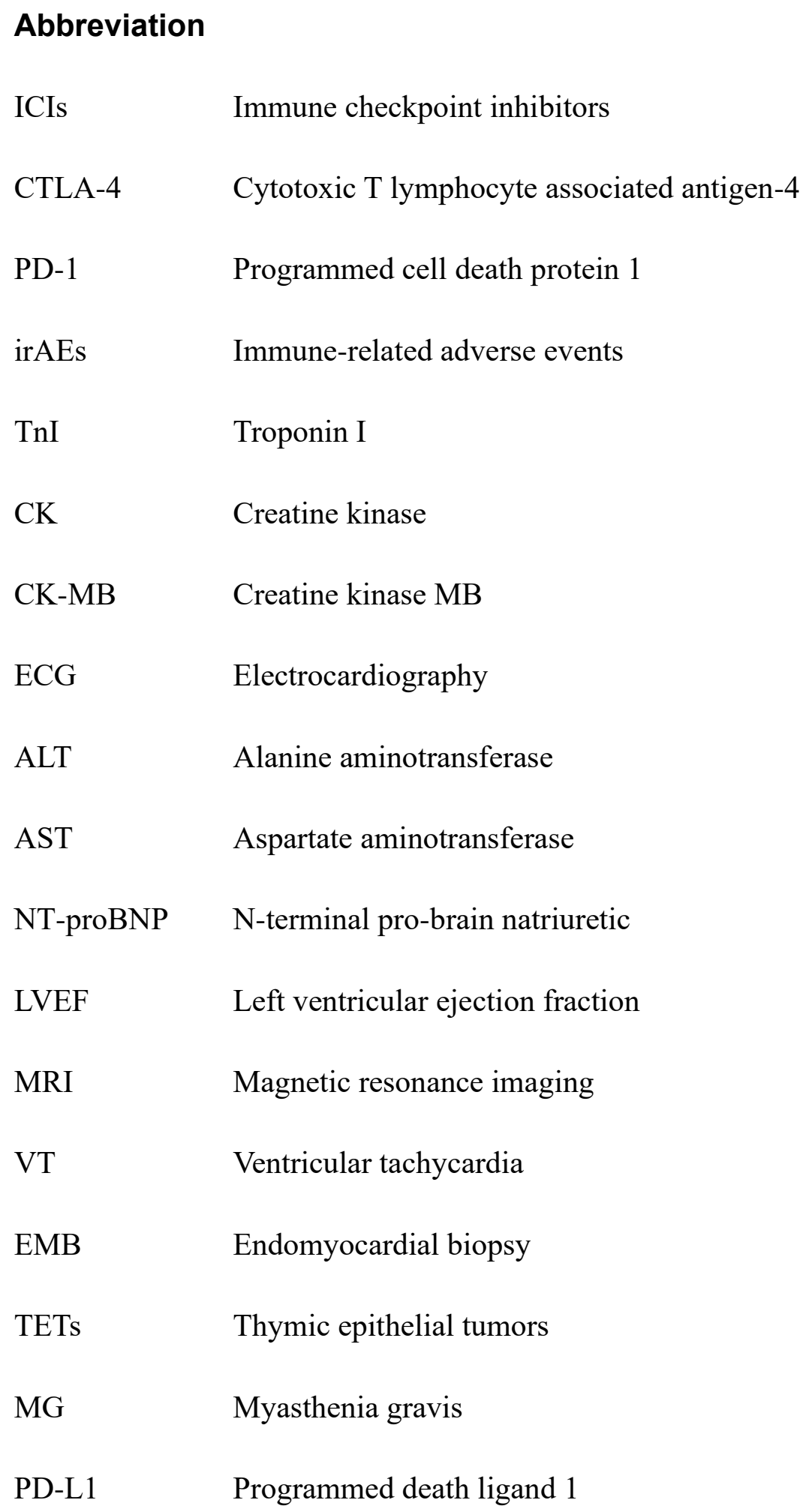




\section{Introduction}

Immune checkpoint inhibitors (ICIs) are widely used for treatment of diverse types of malignances. ICIs block the interaction between cytotoxic $\mathrm{T}$ lymphocyte associated antigen-4 (CTLA-4), programmed cell death protein 1 (PD-1) and their cognate ligands, and thus generate immune responses to tumor cells. However, immune-related adverse events (irAEs) become an inevitable issue, among which colitis, dermatitis, myositis, hepatitis and endocrinopathies are most frequent [1]. Cardiac and neuromuscular irAEs are rare but potentially life-threatening [2]. Here, we report a case with multiple organ damage after the first dose of camrelizumab, a recently approved ICI [3].

\section{Case presentation}

A 68-year-old woman was diagnosed with type B2 thymoma (T4N0M1 stage IV) with left paranephros metastasis in April 2020. The patient was treated with camrelizumab (200 mg, biweekly) as an initial regimen at a nearby hospital. She had no previous history of cardiovascular disease, neurological disorder or any other chronic diseases. Indicators including blood routine examination, transaminase, creatinine, troponin I $(\mathrm{TnI})$, creatine kinase $(\mathrm{CK})$, creatine kinase $\mathrm{MB}(\mathrm{CK}-\mathrm{MB})$, electrocardiography (ECG, Fig. 1a) and echocardiography were unremarkable before initiation of treatment.

However, she was admitted to hospital with complaints of dyspnea, fatigue, 
muscle weakness, palpitation, lightheadedness and poor appetite 11 days following camrelizumab therapy. Vital signs on admission were stable except for increased respiratory rate of 26 breaths/min and heart rate of 120 beats/min. No other positive physical signs were detected. Manual muscle test indicated slight weakness of the arm and leg skeletal muscles. Laboratory studies indicated TnI $13.94 \mathrm{ng} / \mathrm{mL}$ (normal $<0.030$ ), CK-MB 213.70ng/mL (normal <6.30), and CK 8811.40 U/L (normal $<174.0$ ). Alanine aminotransferase (ALT) and aspartate aminotransferase (AST) reached to 290 $\mathrm{U} / \mathrm{L}($ normal <40) and $805 \mathrm{U} / \mathrm{L}$ (normal <35), respectively (Table 1). Plasma D-dimer and N-terminal pro-brain natriuretic peptide increased, with limited clinical significance, to $690.2 \mathrm{ng} / \mathrm{ml}$ (normal $<550.0$ ) and $1169.53 \mathrm{pg} / \mathrm{ml}$ (normal<900.0), respectively. Arterial blood gas analysis indicated normal partial pressure of carbon dioxide and decreased oxygen partial pressure of $76 \mathrm{mmHg}$. Transthoracic echocardiography revealed preserved left ventricular ejection fraction (LVEF) of 65\% with no marked abnormalities of cardiac chamber size and regional ventricular wall motion. ECG on admission showed a new-onset left anterior fascicular branch block (Fig. 1b), followed by bifascicular branch block on the next day (Fig. 1c). Cardiac magnetic resonance imaging (MRI) could not be successfully achieved due to uncontrollably high respiratory rate. In view of the absence of cardiovascular risk factors, statin use, evidence of virus infection and history of ICI administration a few days prior to hospitalization, as well as multiple organ involvement simultaneously, ICI-related myocarditis, myositis and hepatitis were diagnosed. Intravenous methylprednisolone $(80 \mathrm{mg} /$ day $)$ and other supporting treatments were given to the 
patient empirically, since she declined to undergo biopsy of the associated organs.

On day 3 of hospitalization, ECG revealed an intermittent second degree atrioventricular block, with frequent premature atrial contractions, ST segment elevation and poor R-wave progression in precordial leads (Fig. 1d). Considering the elevated biomarkers of myocardial damage and dynamic ECG alterations, coronary angiography was performed to exclude myocardial infarction in spite of atypical clinical presentations, which revealed normal coronary artery anatomy (Fig. 2a, b). Unfortunately, the patient lapsed into a coma with extremely weak respiratory movement but stable hemodynamic status. Emergency blood gas analysis showed fatal carbon dioxide retention with a pressure of $104 \mathrm{mmHg}$. Myasthenic crisis was highly suspected for the underlying disease of thymoma and a rapid progression to life-threatening respiratory failure due to respiratory muscle involvement. Immediately, tracheal intubation and mechanical ventilation were applied. Higher dose of methylprednisolone ( $1 \mathrm{~g} /$ day) was administered and intravenous immunoglobulin (IVIG, 20 g/day) was initiated after neurological consultation. Whereas, third degree artrioventricular block occurred abruptly during the same day (Fig. 1e) and an urgent temporary pacemaker was placed. Although multiple therapeutic measures were administered, clinical status deteriorated after the occurrence of ventricular tachycardia (VT) with heart rate of 230 beats/min on day 4 of hospitalization (Fig. 1f). The patient presented as Adams-Stokes syndrome and direct current defibrillation was undertaken to terminate the fatal tachycardia. However, even though full doses of amiodarone, lidocaine, $\beta$-blocker, sedative and 
corticosteroid were administered intravenously, they failed to relieve the VT storm completely. One day after VT, the patient suffered from ventricular fibrillation followed by cardiac arrest, from which she could not be resuscitated. Postmortem examination was suggested but was refused by her family members.

\section{Discussion}

ICIs are increasingly used in clinical practice, which in turn can potentially activate autoactive $\mathrm{T}$ cells causing immune attack to multiple organs sequentially or simultaneously.

The prevalence of myocarditis caused by several ICIs has been reported previously. Data from Bristol-Myers Squibb corporate safety databases showed a rate of $0.09 \%$ after administration of nivolumab or ipilimumab and a higher rate of $0.27 \%$ when in combination [4]. A multicenter registry reported a prevalence of $1.14 \%$ with a median time of onset of 34 days after starting ICIs [5]. Although ICI-related myocarditis seems to be rare, prognosis can be poor and a mortality rate of $50 \%$ was reported by Salem and colleagues [6]. In the present case, the patient suffered from ICI-related myocarditis with normal LVEF but progressive conduction abnormalities and fatal ventricular arrhythmias secondary to camrelizumab, and this situation may be even more rare [6]. Endomyocardial biopsy (EMB) is the gold standard to diagnose myocarditis, but this method is rarely conducted due to its invasive nature and possible complications. Lymphocytic infiltration within the myocardium, mainly 
positive for $\mathrm{CD} 3$ or the macrophage marker $\mathrm{CD} 68$, is the common pathological presentation of ICI-related myocarditis. Apart from the myocardium, the sinus and atrioventricular nodes can also be infiltrated with lymphocytes, which are potential targets of the activated T cells, so different arrhythmias and conduction abnormalities may occur as a consequence [4]. Cardiac MRI, which is of high sensitivity and specificity and usually considered as a surrogate to EMB to some extent, plays an important role in diagnosis of myocarditis with traditional causes. However, the diagnostic value of this noninvasive technique in ICI-related myocarditis seems to be not so high as in other common types of myocarditis [7]. In the present case, neither EMB nor cardiac MRI were performed owing to some subjective and objective reasons. Even without support of the two aforementioned examinations, it was reasonable to diagnosed ICI-related myocarditis on the basis of recent medication history, new-onset clinical presentations, elevated myocardial damage biomarkers, dynamic ECG changes and exclusion of other confusing causes. Intravenous glucocorticoid was given to the patient immediately after diagnosis of ICI-related myocarditis, but it could not stop or at least slow down the progression of cardiac conduction disturbance.

Thymus is a central immune organ that is responsible for T-cell development by positive and negative selection. Thymic epithelial tumors (TETs) include thymic carcinoma and thymoma. Thymoma is usually accompanied by autoimmune disorders, among which myasthenia gravis (MG) is the most common, with a high rate of $30-44 \%$ [8]. Other immunological disorders such as myositis, myocarditis, colitis, 
hepatitis, lupus erythematosus, and Graves' disease, can also be involved, at relatively lower frequencies. Generally, chemotherapy or chemoradiotherapy is recommended for metastatic thymoma, while unsatisfactory responses are often observed in clinical settings. Studies have shown abundant programmed death ligand 1 (PD-L1) expression using immunohistochemistry in TETs $[9,10]$, which support the potential clinical efficacy of ICIs in TETs. Case series and phase II trials have further demonstrated that ICIs are effective in advanced TETs and thymoma [11-13]. More importantly, the prevalence of ICI-related myocarditis and other irAEs is markedly higher in patients with TETs than with other types of cancers. In one phase II trial, investigating the use of pembrolizumab in patients with recurrent or relapsed TETs, $9.1 \%, 6.1 \%, 12.1 \%$ and $3.0 \%$ of the patients developed myocarditis, MG, hepatitis and thyroiditis, respectively [13]. There is a case report of a fatal immune-related storm in a thymoma patient treated with pembrolizumab, leading to hepatotoxicity accompanied by lymphocytosis, thrombocytopenia and thyroid dysfunction [14]. Multiple organ damage was also involved in our case. Apart from the rapid progression of cardiac adverse events, myasthenic crisis is another fatal complication. The literature suggests that ICI-related MG can be exemplified by de novo presentations and exacerbation of pre-existing or subclinical $\mathrm{MG}$, with a high proportion of $72.7 \%$ for the former [15]. With no MG manifestation prior to administration of camrelizumab, ICI-related de novo MG may be the most likely diagnosis of our patient, although exacerbation of subclinical MG could not be excluded. Immature and TET-derived thymocytes that have escaped self-tolerance 
and become auto-active may be the mechanism for the development of MG in patients with TETs [16]. ICI therapy can further activate $\mathrm{T}$ lymphocytes and strength the autoimmune activity of the cells, given the fact that TET patients are more susceptible to developing autoimmune disorders, which makes thymoma patients more likely than patients with other cancers to experience multiple irAEs $[8,13]$. Even a higher dose of glucocorticoid and IVIG were administered immediately after diagnosis of myasthenic crisis, they could not reverse the outcome due to the extremely worsening situation.

To the best of our knowledge, this is the first report of a thymoma patient developing ICI-related myocarditis, concomitant with de novo $\mathrm{MG}$, myositis and hepatitis, after the first dose of camrelizumab. Some attention should be paid by clinicians to the following: (1) Complete evaluation must be performed before initiation of ICI, and patients already with or at high risk for autoimmune disorders may not be suitable candidates. (2) Sometimes irAEs can be non-specific and even asymptomatic, so close regular follow-up with ECG, echocardiography, and measurement of troponin, $\mathrm{CK}-\mathrm{MB}, \mathrm{CK}$ and transaminases should be conducted within the first week after ICI treatment. (3) Once diagnosed with severe irAEs, high dose of glucocorticoid, mycophenolate mofetil, IVIG, antithymocyte globulin, and plasmapheresis must be applied alone or in combination. (4) irAEs are often characterized by multiple organ involvement, hence a multidisciplinary approach is important for better management of that group of patients. 


\section{References}

1. Thompson JA, Schneider BJ, Brahmer J, Andrews S, Armand P, Bhatia S, Budde LE, Costa L, Davies M,

Dunnington D, Ernstoff MS, Frigault M, Hoffner B, Hoimes CJ, Lacouture M, Locke F, Lunning M, Mohindra NA, Naidoo J, Olszanski AJ, Oluwole O, Patel SP, Reddy S, Ryder M, Santomasso B, Shofer S, Sosman JA, Wahidi M, Wang Y, Johnson-Chilla A, Scavone JL (2019) Management of Immunotherapy-Related Toxicities, Version 1.2019. J Natl Compr Canc Netw 17(3):255-289. https://doi.org/10.6004/jnccn.2019.0013

2. Wang DY, Salem J, Cohen JV, Chandra S, Menzer C, Ye F, Zhao S, Das S, Beckermann KE, Ha L, Rathmell WK, Ancell KK, Balko JM, Bowman C, Davis EJ, Chism DD, Horn L, Long GV, Matteo SC (2018) Fatal Toxic Effects Associated With Immune Checkpoint Inhibitors: A Systematic Review and Meta-analysis. JAMA Oncol. https://doi.org/10.1001/jamaoncol.2018.3923

3. Markham A, Keam SJ (2019) Camrelizumab: First Global Approval. Drugs 79(12):1355-1361. https://doi.org/10.1007/s40265-019-01167-0

4. Johnson DB, Balko JM, Compton ML, Chalkias S, Gorham J, Xu Y, Hicks M, Puzanov I, Alexander MR, Bloomer TL, Becker JR, Slosky DA, Phillips EJ, Pilkinton MA, Craig-Owens L, Kola N, Plautz G, Reshef DS, Deutsch JS, Deering RP, Olenchock BA, Lichtman AH, Roden DM, Seidman CE, Koralnik IJ, Seidman JG, Hoffman RD, Taube JM, Diaz LA Jr, Anders RA, Sosman JA, Moslehi JJ (2016) Fulminant Myocarditis with Combination Immune Checkpoint Blockade. N Engl J Med 375(18):1749-1755. https://doi.org/10.1056/NEJMoa1609214

5. Mahmood SS, Fradley MG, Cohen JV, Nohria A, Reynolds KL, Heinzerling LM, Sullivan RJ, 
Damrongwatanasuk R, Chen CL, Gupta D, Kirchberger MC, Awadalla M, Hassan M, Moslehi JJ, Shah SP, Ganatra S, Thavendiranathan P, Lawrence DP, Groarke JD, Neilan TG (2018) Myocarditis in Patients Treated With Immune Checkpoint Inhibitors. J Am Coll Cardiol 71(16):1755-1764. https://doi.org/ 10.1016/j.jacc.2018.02.037

6. Salem JE, Manouchehri A, Moey M, Lebrun-Vignes B, Bastarache L, Pariente A, Gobert A, Spano JP, Balko JM, Bonaca MP, Roden DM, Johnson DB, Moslehi JJ (2018) Cardiovascular toxicities associated with immune checkpoint inhibitors: an observational, retrospective, pharmacovigilance study. Lancet Oncol 19(12):1579-1589. https://doi.org/10.1016/S1470-2045(18)30608-9

7. Lili Z, Magid A, Mahmood Syed S, Anju N, Hassan Malek Z O, Franck T, Zlotoff Daniel A, Murphy Sean P, Stone James R, Alexandra GDL, Alvi Raza M, Adam R, Maeve J, Cohen Justine V, Heinzerling Lucie M, Connor M, Merna A, Ana B, F.. (2020) Cardiovascular magnetic resonance in immune checkpoint inhibitor-associated myocarditis. Eur Heart J 41(18). https://doi.org/10.1093/eurheartj/ehaa051

8. Lippner EA, Lewis DB, Robinson WH, Katsumoto TR (2019) Paraneoplastic and Therapy-Related Immune Complications in Thymic Malignancies. Curr Treat Options Oncol 20(7):62. https://doi.org/ 10.1007/s11864-019-0661-2

9. Yokoyama S, Miyoshi H (2018) Thymic tumors and immune checkpoint inhibitors. J Thorac Dis 10(Suppl 13):S1509-1509S1515. https://doi.org/10.21037/jtd.2017.10.157

10. Arbour KC, Naidoo J, Steele KE, Ni A, Moreira AL, Rekhtman N, Robbins PB, Karakunnel J, Rimner A, Huang J, Riely GJ, Hellmann MD (2017) Expression of PD-L1 and other immunotherapeutic targets in thymic epithelial tumors. PLoS One 12(8):e0182665. https://doi.org/10.1371/journal.pone.0182665

11. Rajan A, Heery CR, Thomas A, Mammen AL, Perry S, O'Sullivan Coyne G, Guha U, Berman A, Szabo E, Madan RA, Ballester LY, Pittaluga S, Donahue RN, Tsai YT, Lepone LM, Chin K, Ginty F, Sood A, Hewitt 
SM, Schlom J, Hassan R, Gulley JL (2019) Efficacy and tolerability of anti-programmed death-ligand 1

(PD-L1) antibody (Avelumab) treatment in advanced thymoma. J Immunother Cancer 7(1):269.

https://doi.org/10.1186/s40425-019-0723-9

12. Giaccone G, Kim C, Thompson J, McGuire C, Kallakury B, Chahine JJ, Manning M, Mogg R, Blumenschein WM, Tan MT, Subramaniam DS, Liu SV, Kaplan IM, McCutcheon JN (2018) Pembrolizumab in patients with thymic carcinoma: a single-arm, single-centre, phase 2 study. Lancet Oncol 19(3):347-355. https://doi.org/10.1016/S1470-2045(18)30062-7

13. Cho J, Kim HS, Ku BM, Choi YL, Cristescu R, Han J, Sun JM, Lee SH, Ahn JS, Park K, Ahn MJ (2019) Pembrolizumab for Patients With Refractory or Relapsed Thymic Epithelial Tumor: An Open-Label Phase II Trial. J Clin Oncol 37(24):2162-2170. https://doi.org/10.1200/JCO.2017.77.3184

14. Argentiero A, Solimando AG, Ungaro V, Laforgia M, Strippoli S, Pinto D, Negri A, Ferraiuolo S, Zito A, Guida M (2020) Case Report: Lymphocytosis Associated With Fatal Hepatitis in a Thymoma Patient Treated With Anti-PD1: New Insight Into the Immune-Related Storm. Front Oncol 10:583781. https://doi.org/10.3389/fonc.2020.583781

15. Makarious D, Horwood K, Coward J (2017) Myasthenia gravis: An emerging toxicity of immune checkpoint inhibitors. Eur J Cancer 82:128-136. https://doi.org/10.1016/j.ejca.2017.05.041

16. Shahar S, Nancy A, Arie A, Yehuda S (2011) Thymoma and autoimmunity. Cell Mol Immunol 8(3). https://doi.org/10.1038/cmi.2010.74 
Table 1 Biomarkers of myocardial, muscular and hepatic damages

\begin{tabular}{llllllll}
\hline Parameters & $\begin{array}{l}\text { Normal } \\
\text { Range }\end{array}$ & $\begin{array}{l}\text { Before } \\
\text { ICI use }\end{array}$ & $\begin{array}{l}\text { On } \\
\text { admission }\end{array}$ & Day 2 & Day 3 & Day 4 & Day 5 \\
\hline TnI (ng/ml) & $<0.030$ & 0.01 & 13.94 & 36.05 & 63.0 & 46.0 & 20.8 \\
CK-MB & $0.60-6.30$ & 1.10 & 213.70 & 233.6 & 135.1 & 71.2 & 33.8 \\
$(\mathrm{ng} / \mathrm{ml})$ & & & & & & & \\
$\mathrm{CK}(\mathrm{U} / \mathrm{L})$ & $38.0-174.0$ & 20.4 & 8811.4 & 4051.3 & 2245.9 & 1259.5 & 867.9 \\
$\mathrm{ALT}(\mathrm{U} / \mathrm{L})$ & $7-40$ & 12 & 290 & - & 273 & - & 183 \\
AST (U/L) & $13-35$ & 18 & 805 & - & 566 & - & 155 \\
\hline
\end{tabular}

ALT, alanine aminotransferase; AST, aspartate aminotransferase; CK, creatine kinase; CK-MB, creatine kinase MB; TnI, troponin I; ICI, immune checkpoint inhibitor 


\section{Figures:}

Fig 1 Progressive alterations on electrocardiography

(a) Sinus rhythm and normal cardiac conduction before immune checkpoint inhibitor use. (b) New-onset left anterior fascicular branch block on admission. (c) New-onset complete right branch block plus left anterior fascicular branch block, namely bifascicular branch block, on day 2 of hospitalization. (d) Intermittent second degree atrioventricular block, with frequent premature atrial contractions, ST segment elevation and poor R-wave progression in precordial leads on hospital day 3. (e) The third degree atrioventricular block also on hospital day 3. (f) Ventricular tachycardia (VT) with heart rate of $230 / \mathrm{min}$ on hospital day 4.

Fig 2 Coronary angiography showing a normal coronary artery anatomy 


\section{Figures}
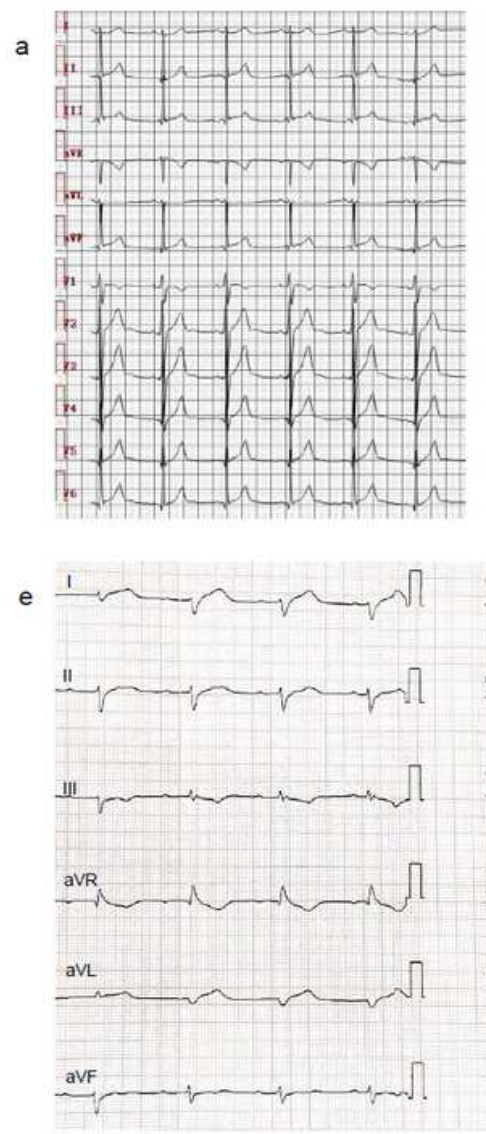
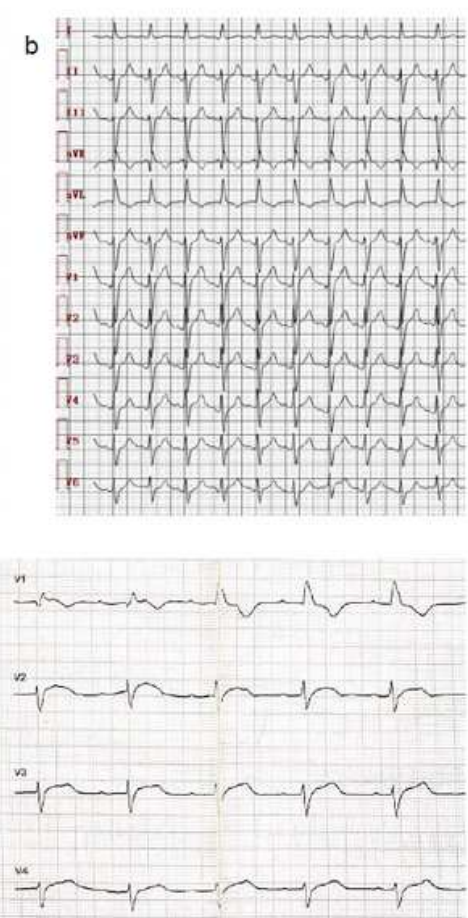

vs

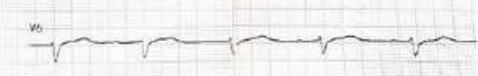

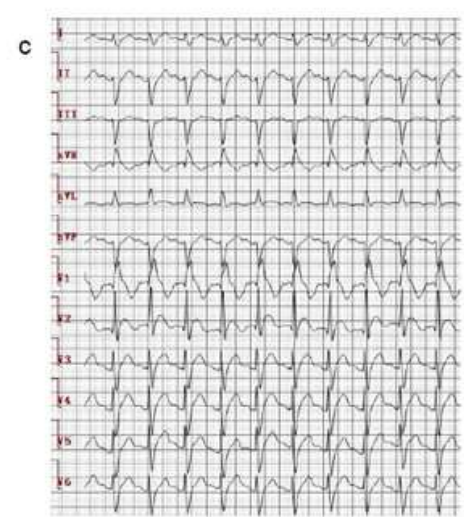
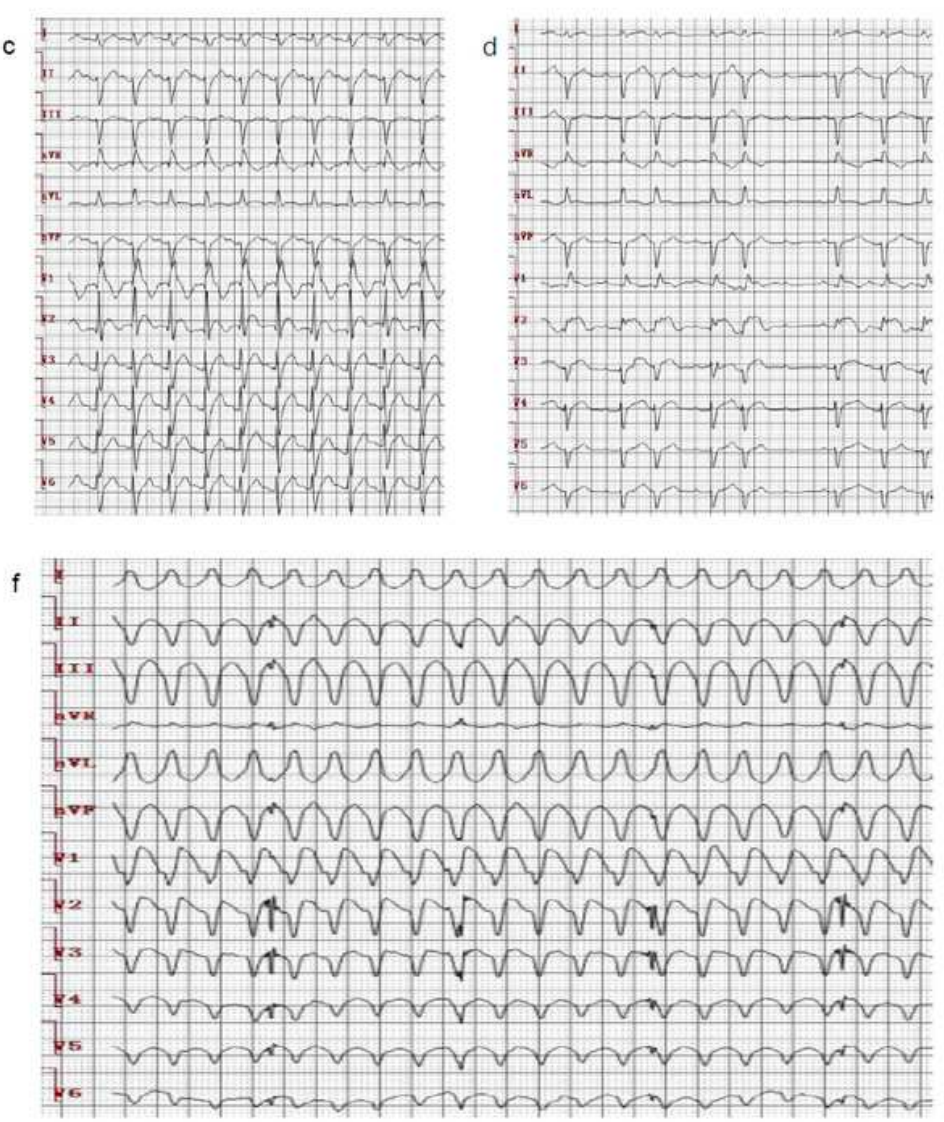

\section{Figure 1}

Progressive alterations on electrocardiography (a) Sinus rhythm and normal cardiac conduction before immune checkpoint inhibitor use. (b) New onset left anterior fascicular branch block on admission. (c) New onset complete right branch block plus left anterior fascicular branch block, namely bifascicular branch block, on day 2 of hospitalization. (d) Intermittent second degree atrioventricular block, with frequent premature atrial contractions, ST segment elevation and poor R wave progression in precordial leads on hospital day 3. (e) The third degree atrioventricular block also on hospital day 3. (f) Ventricular tachycardia (VT) with heart rate of 230 /min on hospital day 4. 
a

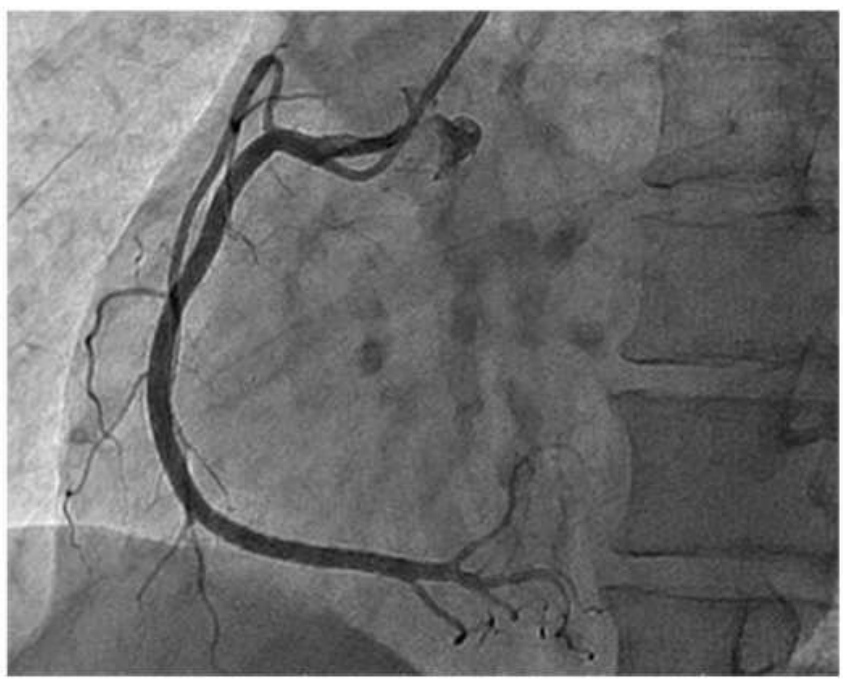

b

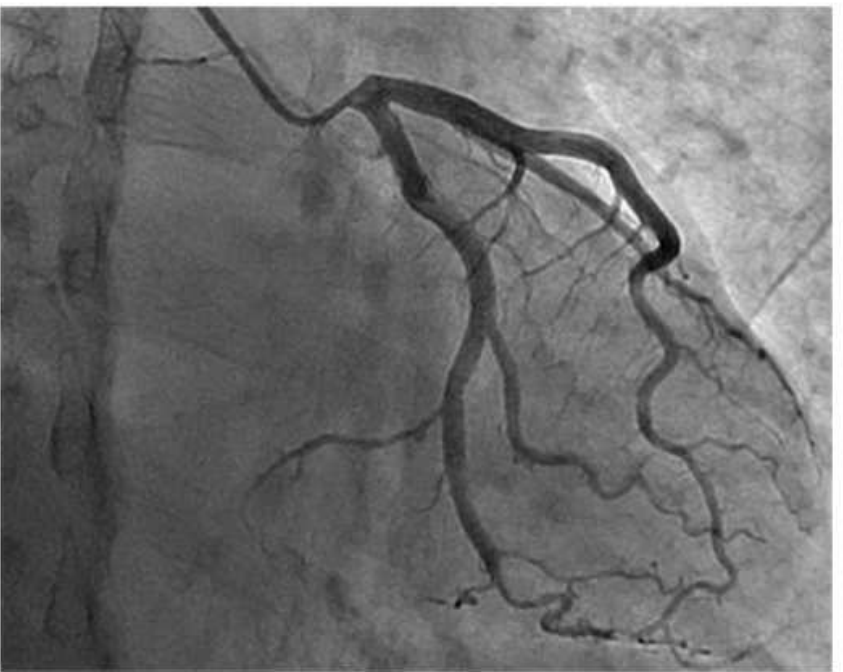

Figure 2

Coronary angiography showing a normal coronary artery anatomy 